\title{
Encapsulation of single-molecule magnets in carbon nanotubes
}

\author{
Maria del Carmen Giménez-López, Fabrizio Moro, Alessandro La Torre', Carlos J. Gómez-García², \\ Paul D. Brown ${ }^{3}$, Joris van Slageren ${ }^{1, \dagger} \&$ Andrei N. Khlobystov ${ }^{1}$
}

Next-generation electronic, photonic or spintronic devices will be based on nanoscale functional units, such as quantum dots, isolated spin centres or single-molecule magnets. The key challenge is the coupling of the nanoscale units to the macroscopic world, which is essential for read and write purposes. Carbon nanotubes with one macroscopic and two nanoscopic dimensions provide an excellent means to achieve this coupling. Although the dimensions of nanotube internal cavities are suitable for hosting a wide range of different molecules, to our knowledge, no examples of molecular magnets inserted in nanotubes have been reported to date. Here we report the successful encapsulation of single-molecule magnets in carbon nanotubes, yielding a new type of hybrid nanostructure that combines all the key single-molecule magnet properties of the guest molecules with the functional properties of the host nanotube. The findings may pave the way to the construction of spintronic or ultrahigh-density magnetic data storage devices.

\footnotetext{
${ }^{1}$ School of Chemistry, University of Nottingham, University Park, Nottingham NG7 2RD, UK. ${ }^{2}$ Instituto de Ciencia Molecular, Universidad de Valencia, Parque Científico, Paterna 46980, Spain. ${ }^{3}$ Division of Materials, Mechanics and Structures, Department of Mechanical, Materials and Manufacturing Engineering, Faculty of Engineering, University of Nottingham, University Park, Nottingham NG7 2RD, UK. †Present address: Institut für Physikalische Chemie, Pfaffenwaldring 55, D-70569 Stuttgart, Germany. Correspondence and requests for materials should be addressed to A.N.K. (email: Andrei.khlobystov@nottingham.ac.uk) or to J.v.S (email: slageren@ipc.uni-stuttgart.de).
} 
S ingle-molecule magnets (SMM) possess a wide range of functional properties unique to this class of materials, including magnetic bistability ${ }^{1}$, quantum tunnelling of magnetization ${ }^{2,3}$, and quantum coherence ${ }^{4,5}$. These properties are unattainable in other types of magnetic molecules, including endohedral fullerenes, such as $\mathrm{N} @ \mathrm{C}_{60}$, which, however, exhibits much longer electron spin relaxation times ${ }^{6-8}$ than those of SMM. The properties of SMM can be exploited for applications in molecular electronics ${ }^{9}$, spintronics $^{10}$, data storage devices ${ }^{11}$ and quantum information processing ${ }^{12}$. However, any practical application of SMM requires their interaction with the macroscopic world, to allow read-and-write processes. A possible approach to this end is deposition of SMM on conductive surfaces, which, however, often leads to SMM decomposition ${ }^{13,14}$. Carbon nanotubes (NT) offer an excellent solution to connecting SMM to the outside world, without attendant problems of deterioration of the SMM functionality. The state of the SMM in such a hybrid NT-SMM material can be controlled electrically, because NT are excellent electrical conductors ${ }^{14}$. Such electrical control is essential for local addressability for data storage, spintronics or quantum information processing applications ${ }^{15,16}$. Furthermore, NT are chemically and thermally very robust, which is highly advantageous for device fabrication ${ }^{17}$. Finally, the nanotube that encapsulates the SMM also protects the guest molecule from the surroundings that would otherwise induce strong decoherence, which is highly detrimental for quantum information processing applications ${ }^{18}$. The magnetic bistability of SMM, essential for data storage applications, derives from a combination of two factors ${ }^{11}$. The intramolecular superexchange interactions give rise to a well-defined ground state with high spin. For example, the ground state of the archetypical SMM, $\left[\mathrm{Mn}_{12} \mathrm{O}_{12}\left(\mathrm{O}_{2} \mathrm{CCH}_{3}\right)_{16}\left(\mathrm{H}_{2} \mathrm{O}\right)_{4}\right]$ or $\mathrm{Mn}_{12}$ Ac for short, has a spin of $S=10$. Secondly, the zero-field splitting parameter $D$ is large and negative, leading to a preferential magnetization direction, the easy axis. These two factors combined result in a large energy barrier for magnetization reversal, which gives rise to slow relaxation of the magnetization and concurrent magnetic bistability.

The shape of $\mathrm{M}_{12} \mathrm{Ac}$ can be described as a discoid with a diameter of $1.6 \mathrm{~nm}$ and a height of $1.1 \mathrm{~nm}$ (Fig. 1a). Considering the minimum van der Waals gap of $0.3 \mathrm{~nm}$ required between the surface of the molecule and the inner surface of $\mathrm{NT}^{17}$, the smallest diameter of NT capable of hosting $\mathrm{Mn}_{12} \mathrm{Ac}$ is $2.2 \mathrm{~nm}$. Therefore,

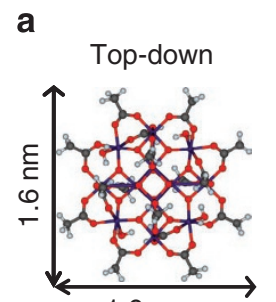

$1.6 \mathrm{~nm}$
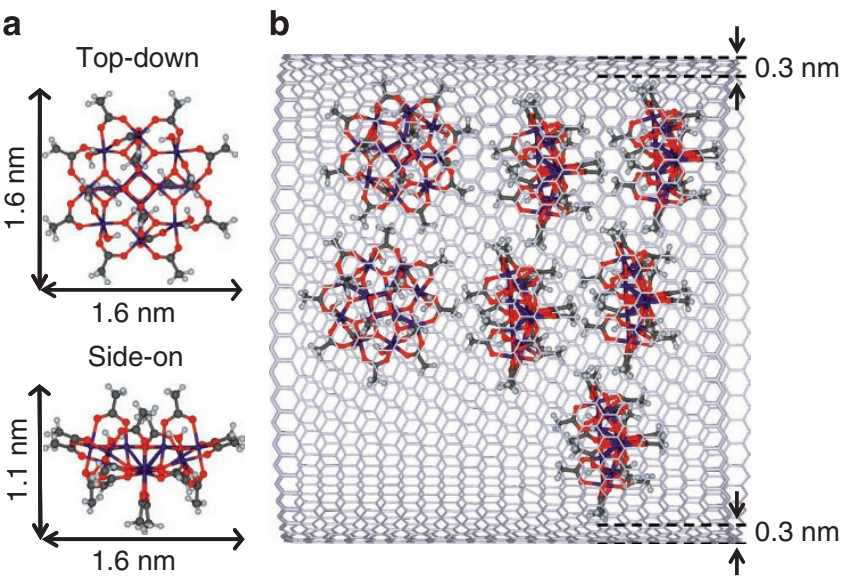

Figure 1 | Chemical structure of $\mathbf{M n}_{12} \mathrm{Ac} S M M$ and schematic representation of its encapsulation in a carbon nanotube. (a) Ball and stick structure of $\left[\mathrm{Mn}_{12} \mathrm{O}_{12}\left(\mathrm{O}_{2} \mathrm{CCH}_{3}\right)_{16}\left(\mathrm{H}_{2} \mathrm{O}\right)_{4}\right]\left(\mathrm{Mn}_{12} \mathrm{Ac}\right)$, with its dimensions indicated by double-headed arrows. Carbon, hydrogen, manganese and oxygen atoms are shown as grey, light-blue, purple and red circles, respectively. (b) Schematic representation of the innermost layer of a GMWNT hosting $\mathrm{Mn}_{12}$ Ac molecules. insertion of $\mathrm{Mn}_{12} \mathrm{Ac}$, as indeed other SMM whose dimensions tend to be even larger, into single-walled carbon nanotubes (SWNT; typical diameter range $1-2 \mathrm{~nm}$ ) is not feasible, and thus wider multiwalled carbon nanotubes (MWNT) (internal diameter range $5-50 \mathrm{~nm}$ ) should be used as host-structures for SMM. Indeed, all previous attempts of combining SMM and SWNT resulted in composite structures where the molecules, adsorbed on the nanotube surface, were exposed to the detrimental influences of the environment (atmospheric oxygen and water, or ambient light), often leading to fast degradation of their magnetic properties ${ }^{19-21}$.

Here we report the preparation and study of a hybrid material in which $\mathrm{Mn}_{12}$ Ac SMM are encapsulated inside MWNT (Fig. 1b). We show that the SMM functional properties are fully retained, and that the molecules undergo a large degree of orientational ordering inside the nanotube. This ordering is advantageous for addressing purposes and for controlling electronic properties of the nanotube.

\section{Results}

Encapsulation of SMM into carbon nanotubes. We chose graphitized MWNT (GMWNT), produced by catalytic chemical vapour deposition at $2,800^{\circ} \mathrm{C}$, with a length of $10-50 \mu \mathrm{m}$, and a mean internal diameter of $6.5 \pm 1.8 \mathrm{~nm}$ (Supplementary Figs S1-S4), because these GMWNT are sufficiently wide for insertion of SMM, and are completely free of residual catalyst that complicated the analysis of the magnetic properties of other nanotube-based structures $^{20}$.

$\mathrm{Mn}_{12} \mathrm{Ac}$ is by far the most-studied SMM and exhibits a blocking temperature as high as $4 \mathrm{~K}$, which is much higher than that of the vast majority of other $\mathrm{SMM}^{11}$. To allow encapsulation of $\mathrm{Mn}_{12} \mathrm{Ac}$ SMM, the GMWNT were pre-treated with concentrated nitric acid followed by heating in air, yielding open GMWNT with an average length of $400 \pm 200 \mathrm{~nm}$. We have used supercritical $\mathrm{CO}_{2}\left(\mathrm{scCO}_{2}\right)$ for the transport of the SMM molecules into the nanotubes. $\mathrm{scCO}_{2}$ is an excellent carrier fluid for the encapsulation of molecules, because the small size of $\mathrm{CO}_{2}$, its low viscosity, high diffusivity and zero surface tension allow it to penetrate the nanotubes without hindrance, enabling insertion of the desired guest species ${ }^{22-24}$. The mild filling conditions, with temperatures not exceeding $40^{\circ} \mathrm{C}$, allowed for the successful insertion of $\mathrm{Mn}_{12} \mathrm{Ac}$, which is far too fragile to be transported into GMWNT through the gas or molten phase. The resulting hybrid material, Mn $\mathrm{n}_{12}$ Ac@GMWNT (1), was carefully washed with acetonitrile (a good solvent for $\mathrm{Mn}_{12} \mathrm{Ac}$ ) to eliminate any $\mathrm{Mn}_{12} \mathrm{Ac}$ molecules outside the NT (Supplementary Figs S5 and S6).

The nanotubes in material $\mathrm{Mn}_{12}$ Ac@GMWNT (1), as observed by transmission electron microscopy (TEM), comprise discrete, free-standing entities (Fig. 2a). There is no indication of $\mathrm{Mn}_{12} \mathrm{Ac}$ aggregates outside the NT in $\mathrm{Mn}_{12}$ Ac@GMWNT, whereas in a control sample before washing (Supplementary Fig. S7), these aggregates can be clearly seen. The higher magnification micrograph (Fig. 2b) shows a series of parallel lines corresponding to the sidewalls of the GMWNT, and dark contrast attributable to clusters of individual $\mathrm{Mn}_{12} \mathrm{Ac}$ molecules positioned within the GMWNT cavity (Fig. 2d). Indeed, energy-dispersive X-ray analysis (EDX) spectra taken from filled nanotubes using a $4 \mathrm{~nm}$ e-beam probe unequivocally prove the presence of manganese inside the nanotubes (Fig. 2c). It is noted that $\mathrm{Mn}_{3} \mathrm{O}_{4}$, an expected decomposition product of $\mathrm{Mn}_{12} \mathrm{Ac}$, which would exhibit a characteristic crystal lattice structure in TEM, was not observed in this sample (Supplementary Fig. S8), thus confirming that intact $\mathrm{Mn}_{12} \mathrm{Ac}$ molecules are present inside the nanotubes, consistent with the magnetic measurements described below. Under the influence of the e-beam in the TEM, the $\mathrm{Mn}_{12} \mathrm{Ac}$ was observed to move within the nanotube cavity (Supplementary Fig. S9), indicating that the molecules are held by van der Waals forces only, with no covalent bonds formed between the SMM and the nanotube sidewalls. 

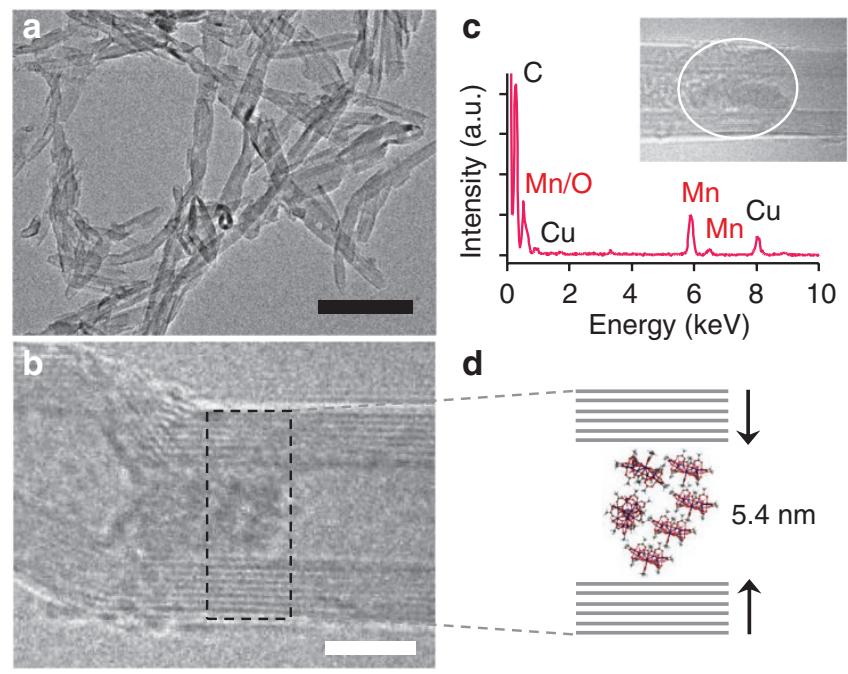

Figure 2 | Structural and compositional characterization of $\mathbf{M n}_{12} \mathbf{A c} @ \mathbf{G M W N T}$ ( $\left.\mathbf{a}, \mathbf{b}\right)$ Conventional bright field and phase contrast transmission electron micrographs of GMWNT filled with $\mathrm{Mn}_{12} \mathrm{Ac}$ (1) (black scale bar is $100 \mathrm{~nm}$ white scale bar is $5 \mathrm{~nm}$ ). (c) EDX spectrum of the selected area in the GMWNT (inset, circled), confirming the presence of Mn-containing molecules within the nanotube (Cu peaks are due to a copper TEM support grid). (d) Schematic representation of the packing of $\mathrm{Mn}_{12} \mathrm{Ac}$ molecules in a nanotube.
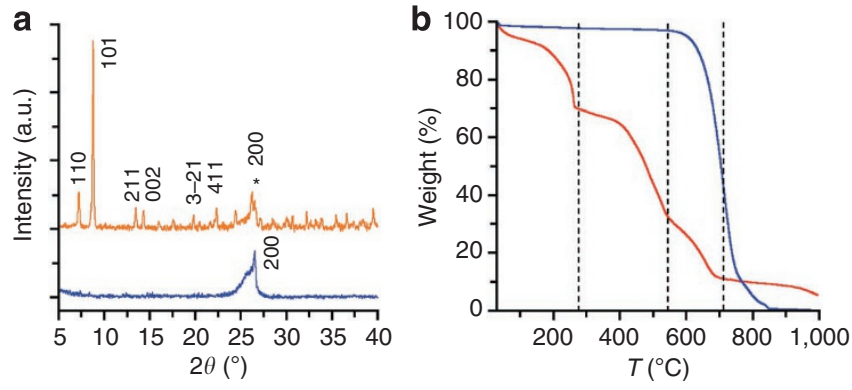

Figure 3 | Preferential interactions of SMM with GMWNT interior revealed through control experiments. Powder X-ray diffractograms (a) of the composite material where $\mathrm{Mn}_{12} \mathrm{Ac}$ is adsorbed on the surface of closed (untreated) GMWNT before (orange) and after (blue) washing with acetonitrile, confirming the efficient removal of $\mathrm{Mn}_{12} \mathrm{Ac}$ molecules from the nanotube surface. The peak labelled with an asterisk is assigned to the nanotube. (b) TGA of $\mathrm{Mn}_{12} \mathrm{Ac} @ G M W N T$ (1) (red) and closed GMWNT after washing (blue).

Our encapsulation procedure combined with a washing step with acetonitrile ensures that all SMM are inside nanotubes, and that no molecules remain on the nanotube surface. A series of rigorous control experiments involving untreated (sealed) GMWNT were performed to ascertain the efficacy of the washing procedure, using exactly the same conditions as for the preparation of $\mathrm{Mn}_{12} \mathrm{Ac} @$ GMWNT (1) (Supplementary Fig. S10). The composite of $\mathrm{Mn}_{12} \mathrm{Ac}$ with sealed GMWNT showed a clear signature in the powder $\mathrm{X}$-ray diffractogram attributable to the presence of $\mathrm{Mn}_{12}$ Ac clusters adsorbed on GMWNT surface, before washing (Fig. 3a-orange). After washing, the only feature remaining in the diffractogram corresponded to the atomic lattice of sealed GMWNT (Fig. 3ablue). Thermogravimetric analysis (TGA) of the sealed GMWNT after washing showed that no metal-containing material remained after heating above $600^{\circ} \mathrm{C}$ (Fig. $3 \mathrm{~b}$-blue). Thus, both X-ray diffraction and TGA data indicate that all the $\mathrm{Mn}_{12} \mathrm{Ac}$ molecules have been removed from the nanotube surface in the washing step. In contrast, the hybrid material $\mathrm{Mn}_{12} \mathrm{Ac} @$ GMWNT (1) subjected to the same washing procedure did not lose its guest $\mathrm{Mn}_{12} \mathrm{Ac}$ molecules, as confirmed by TGA (Fig. 3b-red). These experiments demonstrated that $\mathrm{Mn}_{12} \mathrm{Ac}$ interacts stronger with the interior of GMWNT than with its exterior because of a greater contact area of the discshaped molecule with the concave side as compared with the convex side of the nanotube cylinder, which ensures effective encapsulation of SMM into GMWNT driven by van de Waals force.

The weight loss of the $\mathrm{Mn}_{12}$ Ac@GMWNT (1) in TGA proceeded in three distinct steps (Fig. 3b-red). The first step corresponded to decomposition of $\mathrm{Mn}_{12} \mathrm{Ac}$ with the loss of all coordinated ligands and, hence, the consequent formation of $\mathrm{Mn}_{3} \mathrm{O}_{4}$. The second step corresponded to the catalytic oxidation of the GMWNTs in the presence of the $\mathrm{Mn}_{3} \mathrm{O}_{4}$ formed inside the nanotubes, and the third step corresponded to thermal oxidation of the GMWNT themselves (that is, sections of nanotubes that are not in direct contact with $\left.\mathrm{Mn}_{3} \mathrm{O}_{4}\right)^{25,26}$. The residual weight remaining at $1,000^{\circ} \mathrm{C}$ was attributed to $\mathrm{Mn}_{3} \mathrm{O}_{4}$ that does not decompose further. The first step was also observed in the TGA of pristine, unconfined $\mathrm{Mn}_{12}$ Ac (Supplementary Fig. S11), thus supporting the fact that the $\mathrm{Mn}_{12} \mathrm{Ac}$ molecules remain structurally intact upon insertion into the GMWNT (Supplementary Figs. S12-S17; Supplementary Tables S1-S3).

Differential scanning calorimetry (DSC) measurements showed essentially the same $\mathrm{Mn}_{12} \mathrm{Ac}$ thermal behaviour before and after treatment with $\mathrm{ScCO}_{2}$ (Supplementary Figs S12 and S13), again confirming that the molecules are transported and encapsulated in the GMWNT in their intact form. It is interesting to note that $\mathrm{Mn}_{12}$ Ac molecules inside the nanotubes decompose at a slightly higher temperature $\left(\mathrm{ca} .240^{\circ} \mathrm{C}\right)$ as compared with free molecules (ca. $220^{\circ} \mathrm{C}$ ). This indicates that the confinement of SMM within the nanoscopic cavities of GMWNT enhances their thermal stability and resistance to oxidation in air. These findings demonstrate that $\mathrm{Mn}_{12}$ Ac@GMWNT (1) fabricated in supercritical fluid is a clean, well-defined nanostructured material, with all of the SMM contained inside the nanotubes.

Magnetic behaviour of SMM in nanotubes. The magnetic properties of SMM inserted in nanotubes have been extensively investigated in our study (Fig. 4). A control sample of free, non-encapsulated $\mathrm{Mn}_{12} \mathrm{Ac}$ (2), treated with $\mathrm{scCO}_{2}$ under the same conditions as used for the preparation of $\mathrm{Mn}_{12} \mathrm{Ac} @ G M W N T$ (1), was also studied to ascertain the influence of nanotubes on SMM properties (Supplementary Figs S16 and S17). Alternating current (AC) magnetic susceptibility measurements recorded for $\mathrm{Mn}_{12}$ Ac@GMWNT (1) (Fig. 4a,b) show an out-of-phase $\left(\chi^{\prime \prime}\right)$ signal (Fig. $4 \mathrm{~b}$ ) that is a manifest signature of slow relaxation of magnetization. Furthermore, both the in-phase $\left(\chi^{\prime}\right)$ and out-of-phase $\left(\chi^{\prime \prime}\right)$ signals are strongly frequency dependent, indicating that the observed slow relaxation is due to the SMM behaviour, that is, the presence of an energy barrier for relaxation of the magnetic moment. It is worth noting that the observed frequency dependence of the AC signals follows an Arrhenius law, $\tau=\tau_{0} \exp \left(\Delta E / k_{\mathrm{B}} T\right)$, where $\tau$ is the relaxation time of magnetization, $T$ temperature, $\Delta E$ activation energy and $k_{\mathrm{B}}$ Boltzmann constant, which allows calculation of the energy barrier $\left(\Delta E / k_{\mathrm{B}}\right)$ for the relaxation process of the magnetization of molecular magnets in nanotubes (Supplementary Fig. S19). Importantly, these AC magnetic susceptibility measurements confirmed that the $\mathrm{Mn}_{12} \mathrm{Ac}$ molecules remain intact inside the nanotube, with their functional magnetic properties fully preserved (for $\mathrm{Mn}_{3} \mathrm{O}_{4}$, the maxima in $\chi^{\prime \prime}$ would occur at much higher temperatures, and would not show such a pronounced frequency dependence). To our knowledge, this is the first example of intact SMM that have been encapsulated 

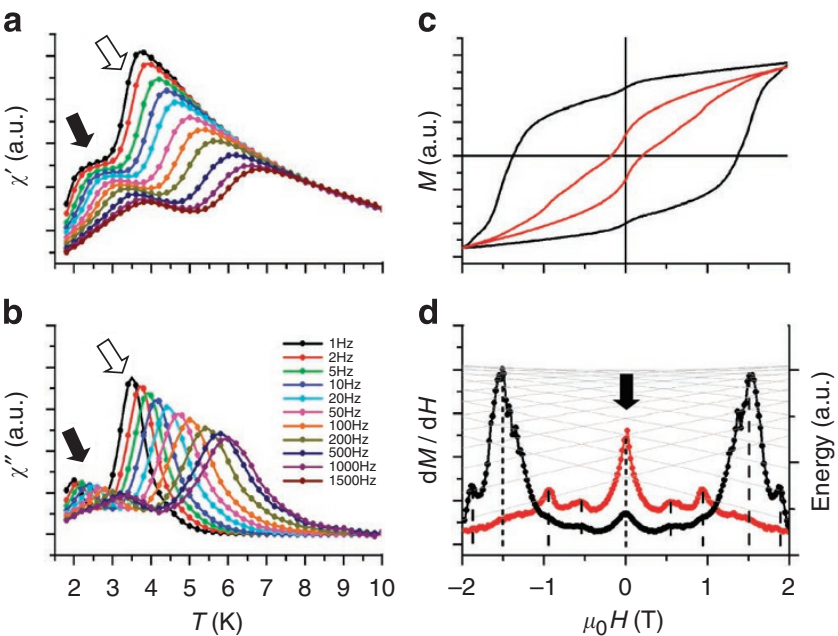

Figure 4 | Magnetic characterization of $\mathbf{M n}_{\mathbf{1 2}} \mathbf{A c} @$ GMWNT. In-phase

(a) and out-of-phase (b) components of the AC susceptibility of $M_{12} A c @$ GMWNT (1) measured with an AC field of 3.50 e at different frequencies, showing slow relaxation of the magnetization of the encapsulated $M_{12} A c$ molecules. (c) Hysteresis curves of magnetization recorded for $M_{12} A c @$ GMWNT (red) at $1.8 \mathrm{~K}$ and for the control sample $\mathrm{Mn}_{12} \mathrm{Ac}$ (2) (black). (d) Derivatives of the hysteresis curves shown in (c), including the energy level scheme for $\mathrm{Mn}_{12}$ Ac plotted as a function of field (thin crossing lines), calculated using parameters reported previously ${ }^{11}$.

inside nanotubes. In all previous studies, SMM mixed with SWNT were adsorbed on the external nanotube surfaces ${ }^{19-21}$. Although surface deposition of $\mathrm{Mn}_{12} \mathrm{Ac}$ is known to cause decomposition of the delicate magnetic molecules ${ }^{13}$, our procedure for the transport and encapsulation of SMM into GMWNT has no detrimental effects on the structural integrity and magnetism of the $\mathrm{Mn}_{12} \mathrm{Ac}$.

Both $\chi^{\prime}$ and $\chi^{\prime \prime}$ display two distinct maxima (labelled with arrows, Fig. 4a,b), attributable to the presence of two slightly different $\mathrm{Mn}_{12} \mathrm{Ac}$ species, with different values of zero-field splitting parameter, $D$. The lower-temperature maximum corresponds to a fast-relaxing species in which Jahn-Teller elongation axis of one of the $\mathrm{Mn}^{3+}$ centres has been tilted by $90^{\circ}$ compared with the normal $\mathrm{Mn}_{12}$ Ac molecule (slow-relaxing species) ${ }^{27}$. For Mn $\mathrm{Mn}_{12} \mathrm{Ac} @$ GMWNT (1), the fraction of fast-relaxing species, which is determined from the intensity of the low-temperature peak, is 20\% compared with $7 \%$ for the control sample $\mathrm{Mn}_{12} \mathrm{Ac}$ (2) (Supplementary Fig. S16 and Supplementary Table S4). This type of Jahn-Teller isomerization has also been observed previously for $\mathrm{Mn}_{12} \mathrm{Ac}$ aggregated in small clusters embedded in polymer matrices ${ }^{28,29}$.

The SMM in nanotubes exhibit magnetic hysteresis at $1.8 \mathrm{~K}$ with sizeable coercivity of $0.2 \mathrm{~T}$ (Fig. 4c), again demonstrating that their properties remain largely unaffected by nanoscale confinement. Step-like features in the hysteresis curves, which become more evident in the plots of numerical derivatives $\mathrm{d} M / \mathrm{d} H$ (Fig. $4 \mathrm{~d}$ ), are attributed to quantum tunnelling of the magnetization. The tunnelling occurs for each longitudinal field at levels $\left(M_{\mathrm{S}}\right.$ states $)$ on both sides of the magnetization relaxation energy barrier that are in resonance ${ }^{2,3}$. These steps (dotted lines, Fig. 4d) occur at the same fields for both $\mathrm{Mn}_{12}$ Ac@GMWNT (1) and the control sample $\mathrm{Mn}_{12} \mathrm{Ac}$ (2), showing both samples to have the same $D$ values. The observed variation of the step positions with the strength of magnetic field correlates well with the crossing energy levels (thin lines, Fig. 4d) calculated for $\mathrm{Mn}_{12} \mathrm{Ac}$ (ref. 11). These observations are consistent with a significant fraction of the molecules becoming oriented with their easy axes aligned parallel to the applied magnetic field. Without such orientation the longitudinal component of the applied field
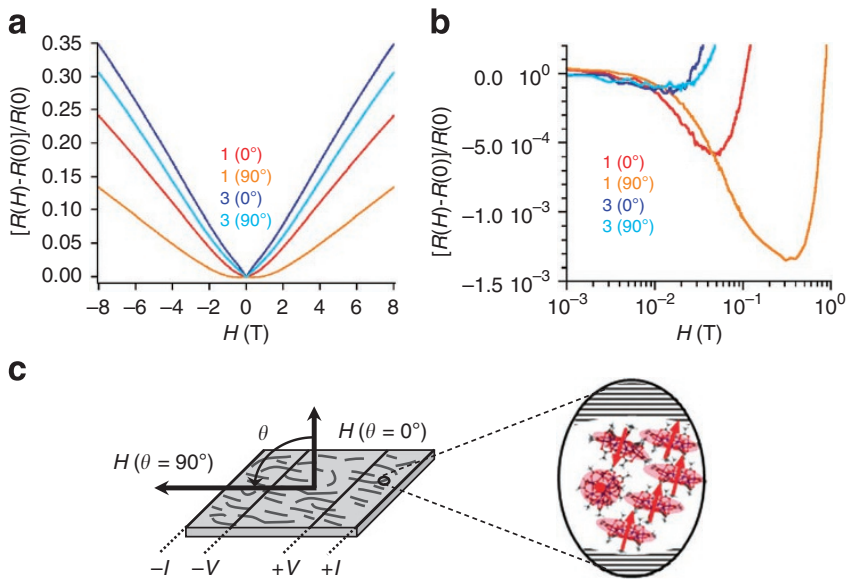

Figure 5 | Magnetoresistance measurements of nanotubes hosting $\mathbf{M n}_{12} \mathbf{A c}$ SMM. (a) Magnetoresistance of $\mathrm{Mn}_{12} \mathrm{Ac} @ G M W N T$ (1) and the control sample of empty (open and short) GMWNT (3) at $2 \mathrm{~K}$ with magnetic field $(\mathrm{H})$ either perpendicular $\left(\theta=0^{\circ}\right)$ or parallel $\left(\theta=90^{\circ}\right)$ to the conducting layers. (b) Low field region of the magnetoresistance measurements on the logarithmic scale. (c) Schematic representation of the device used for magnetoresistance measurements. Inset: schematic representation of the preferential alignment of $\mathrm{Mn}_{12} \mathrm{Ac}$ molecules in the internal cavity of nanotube with respect to the magnetic field.

for each molecule would be different resulting in a smearing-out of the magnetization steps.

The derivative peaks of the magnetization steps near zero field (labelled with a black arrow, Fig. 4d) are due to relaxation of the fastrelaxing species, the magnetization of which is not blocked at this temperature. The relative step size found for $\mathrm{Mn}_{12} \mathrm{Ac@GMWNT} \mathrm{(1)}$ is higher than for $\mathrm{Mn}_{12} \mathrm{Ac}$ (2), indicating more efficient quantum tunnelling and, hence, larger transverse anisotropy of the confined magnetic molecules. The lower coercitivity value in the hysteresis curve for $\mathrm{Mn}_{12}$ Ac@GMWNT (1) (Fig. 4c) with respect to the control sample $\mathrm{Mn}_{12} \mathrm{Ac}$ (2) also confirms the shorter spin relaxation time for the confined $\mathrm{Mn}_{12} \mathrm{Ac}$ inside GMWNT. This observation is also in agreement with the calculated energy barrier for $\mathrm{Mn}_{12} \mathrm{Ac@}$ GMWNT (1) $\left(\Delta E / k_{\mathrm{B}}=57 \pm 1 \mathrm{~K}\right)$ that appears to be lower than for $\mathrm{Mn}_{12} \operatorname{Ac}(2)\left(\Delta E / k_{\mathrm{B}}=72 \pm 4 \mathrm{~K}\right)$.

Magnetoresistance of $\mathbf{M n}_{12} \mathbf{A c @ G M W N T}$. Understanding the response of electric properties of $\mathrm{Mn}_{12} \mathrm{Ac} @ G M W N T$ to applied magnetic field is crucial for future applications of this material in nano-electronic devices. The influence of a magnetic field applied parallel $\left(\theta=90^{\circ}\right)$ or perpendicular $\left(\theta=0^{\circ}\right)$ to a layer of $\mathrm{Mn}_{12} \mathrm{Ac} @$ GMWNT (1) on the resistance of the nanotubes filled with the magnetic molecules has been evaluated, and compared with a control sample of empty (short and open) GMWNT (3), the same as used for the preparation of $\mathrm{Mn}_{12}$ Ac@GMWNT (1). Both samples $\mathrm{Mn}_{12}$ Ac@GMWNT (1) and GMWNT (3) exhibited high roomtemperature-specific conductivities of $c a .20 \mathrm{~S} \mathrm{~cm}^{-1}$, which decrease with temperature (Supplementary Figs S20 and S21) due to intertube contact resistance ${ }^{30,31}$. This indicates that the nanotubes filled with SMM molecules retain their intrinsic electric properties.

The effect of the magnetic field on conductance of $\mathrm{Mn}_{12} \mathrm{Ac} @$ GMWNT became measurable only at temperatures below the blocking temperature of the single-molecule magnet $\mathrm{Mn}_{12} \mathrm{Ac}(c a .5 \mathrm{~K}$, Supplementary Fig. S22) residing in nanotubes. For example, at $2 \mathrm{~K}$ and the field above $1 \mathrm{~T}$, all samples exhibited a simple positive magnetoresistance, regardless of the direction of the applied magnetic field (Fig. 5a). However, at lower fields (below $1 \mathrm{~T}$ ), the magnetoresistance of empty nanotubes and nanotubes filled with SMM 
arequalitativelydifferent(Fig.5b).The sample $\mathrm{Mn}_{12}$ Ac@GMWNT(1) exhibits a significant negative magnetoresistance in this range, which is in a sharp contrast to the control sample GWMNT (3) (Fig. 5b; Supplementary Fig. S23). Another important effect of the magnetic guest molecules is that they change the response of the host nanotubes to the direction of the applied field. For example, magnetoresistance of $\mathrm{Mn}_{12} \mathrm{Ac} @$ GMWNT (1) becomes more negative and its minimum shifts substantially as the field changes from perpendicular $\left(\theta=0^{\circ}\right)$ to parallel $\left(\theta=90^{\circ}\right)$, whereas for empty nanotubes (3) both the depth of the minimum and its position remain unchanged.

The effects of the SMM on nanotubes can be also observed at higher magnetic field ( $>1 \mathrm{~T}$, Fig. 5a). The gradient of the positive magnetoresistance of $\mathrm{Mn}_{12} @$ GMWNT (1) changes by $50 \%$ as the directions of the field changes, whereas the control sample of GMWNT (3) is significantly less sensitive showing only $10 \%$ change. Encapsulated SMM modify the susceptibility of the resistance of nanotubes to the angle of the magnetic field, which relates to a preferential orientation of the $\mathrm{Mn}_{12} \mathrm{Ac}$ molecules in the internal cavities of the nanotubes (Fig. $5 \mathrm{c}$ ). As the $\mathrm{Mn}_{12} \mathrm{Ac}$ have a shape of a disc, with the easy magnetic axis perpendicular to the disc, this magnetic anisotropy of the guest molecules translates into the anisotropic magnetoresistance behaviour of the host nanotubes.

\section{Discussion}

This study describes a methodology for integration of SMM into the internal cavity of carbon nanotubes. The non-covalent interactions, which are responsible for efficient transport and encapsulation of the guest molecules into nanotubes, allow the molecules to stay mobile within the nanotube and align along the applied magnetic field. Although the encapsulated $M_{12}$ Ac molecules retain their SMM properties, the host nanotubes provide an alternative pathway for relaxation of SMM magnetization. In turn, the magnetic guest molecules influence the electrical resistance of the host nanotubes. Therefore, the new class of hybrid structures SMM@GMWNT represents a synergistic material combining the functional properties of nanotubes and magnetic molecules.

Encapsulation of SMM into carbon nanotubes offers a number of exciting opportunities for the applications of these materials in nano-electronics. For example, electrical conductivity of the nanotube that could be precisely controlled by the magnetic states of SMMs can provide a method for generating spin-polarized currents at the nanoscale, which are important for the emerging area of spintronic devices. Insertion of SMMs into carbon nanotubes may enable integration of these molecular materials into ultrasensitive magnetic devices, such as a nanoscale superconducting quantum interference device (nano-SQUID) or a magnetic force microscopy probe, which will open a pathway for harnessing functional properties of SMMs at a single-molecule level-a long-standing challenge in molecular nanomagnetism ${ }^{32}$.

\section{Methods}

Preparation of materials. $\mathrm{Mn}_{12} \mathrm{Ac}$ was synthesized following the procedure described previously ${ }^{33}$. All reagents and solvents were purchased from Sigma-Aldrich and used without further purification. GMWNT (catalysed chemical vapour deposition) were purchased from CheapTubes. GMWNT were pre-treated to shorten and open them, and to enable the encapsulation of $\mathrm{Mn}_{12} \mathrm{Ac}$ molecules.

\section{Preparation of short and open GMWNT via oxidative cutting. GMWNT} $(200 \mathrm{mg})$ were added to a $16 \mathrm{M}$ solution of nitric acid $(50 \mathrm{ml})$ and the resulting black suspension was treated with ultrasound in a water bath for $30 \mathrm{~min}$ at room temperature. The suspension was then heated in air under reflux for $5 \mathrm{~h}$. The resulting mixture was then diluted with deionized water $(200 \mathrm{ml})$, filtered using a $0.45 \mu \mathrm{m}$ pore size PTFE membrane filter, washed thoroughly with water until neutral $\mathrm{pH}$ and ethanol $(50 \mathrm{ml})$ and dried under vacuum to yield a black solid $(192 \mathrm{mg})$. The product was then placed in an alumina crucible and heated in air at $700{ }^{\circ} \mathrm{C}$ until weight loss of $80 \%$ was achieved.

Encapsulation of $\mathbf{M n}_{12} \mathbf{A c}$ in GMWNT. Freshly annealed GMWNT (10 mg, short and open) were added to a suspension of $\mathrm{Mn}_{12} \mathrm{Ac}(30 \mathrm{mg})$ in hexane ( $8 \mathrm{ml}$ ), and the resulting black suspension was transferred into a $10 \mathrm{ml}$ autoclave that was topped up with supercritical carbon dioxide $\left(40^{\circ} \mathrm{C}, 275 \mathrm{bar}\right)$. The pressure was cycled between 270 bar and 120 bar for $20 \mathrm{~h}$. The resulting black solid was then washed with $250 \mathrm{ml}$ acetonitrile and filtered using $0.2 \mu \mathrm{m}$ pore size PTFE membrane filter to give $\mathrm{Mn}_{12} \mathrm{Ac} @$ GMWNT (1).

Control measurements. A series of rigorous control experiments involving untreated (sealed) GMWNT were performed to ascertain the efficacy of the washing procedure, and therefore, less effective interactions of $\mathrm{Mn}_{12} \mathrm{Ac}$ molecules with the outer surface of the nanotubes as compared with the interior of GMWNT (Supplementary Figs S7 and S10).

To confirm that $\mathrm{Mn}_{12} \mathrm{Ac}$ molecules are transported and encapsulated in the GMWNT in their intact form, a control sample of $\mathrm{Mn}_{12} \mathrm{Ac}$ (2) treated with $\mathrm{scCO}_{2}$ under the same conditions as used for preparation of $\mathbf{1}$ was carefully analysed by different analytical techniques all supporting the fact that structure and properties of $\mathrm{Mn}_{12} \mathrm{Ac}$ remain unchanged (Supplementary Figs S11-S17 and Supplementary Tables S1-S3).

To prove that the $\mathrm{Mn}_{12} \mathrm{Ac}$ remains intact inside NT, the thermal properties of $\mathrm{Mn}_{12} \mathrm{Ac} @ G M W N T$ (1) were characterized by DSC and TGA. Additionally, a $\mathrm{Mn}_{12} \mathrm{Ac} @$ GMWNT sample heated at $340^{\circ} \mathrm{C}$ was analysed by TEM (Supplementary Figs S24 and S25), revealing that $\mathrm{Mn}_{3} \mathrm{O}_{4}$ is formed as a product of thermal decomposition of $\mathrm{Mn}_{12} \mathrm{Ac}$ in GMWNT.

To verify that Mn-peaks in the EDX are indeed due to $\mathrm{Mn}_{12} \mathrm{Ac}$ guest molecules, EDX spectra were taken for empty sections of nanotubes in $\mathrm{Mn}_{12}$ Ac@GMWNT and the supporting carbon film, none of which showed any indication of Mn (Supplementary Fig. S26).

Analytical techniques. TGA measurements were carried out on a SDT Q-600 TA instrument over the range $25-1,000^{\circ} \mathrm{C}$ in air and at a scan rate of $5{ }^{\circ} \mathrm{C} \mathrm{min}^{-1}$. DSC measurements were carried out on a Mettler Toledo DSC 821e over the range $25-500^{\circ} \mathrm{C}$ with a scan rate of $5^{\circ} \mathrm{C} \mathrm{min}^{-1}$ in air or nitrogen. X-ray powder diffraction measurements were performed on a PANalytical X'Pert PRO diffractometer equipped with a $\mathrm{Cu} \mathrm{K} \alpha$ radiation source $(\lambda=1.5418 \AA)$ in Bragg-Brentano geometry using a Si zero-background holder. Infrared spectra were measured as $\mathrm{KBr}$ discs on a Nicolet Avatar 380 FTIR spectrometer over the range $400-4,000 \mathrm{~cm}^{-1}$. Elemental analyses were performed by the Elemental Analysis Service of London Metropolitan University. Mass spectrometry was carried out on a Bruker ApexIV with electro spray ionization.

Transmission electron microscopy characterization. High-resolution TEM imaging and energy-disperse X-ray analyses were performed using a Jeol 2100F transmission electron microscope using an accelerating voltage of $100 \mathrm{kV}$. TEM samples were prepared by casting several drops of hexane suspension of $\mathrm{Mn}_{12} \mathrm{Ac} @$ GMWNT onto copper-grid mounted 'lacey' carbon films before drying under a stream of nitrogen. Statistical analysis of TEM images were performed using Gatan Digital Micrograph software.

Magnetic measurements. A commercial Quantum Design MPMS-XL5 SQUID magnetometer was used for the magnetic characterization of the samples: $\mathrm{Mn}_{12} \mathrm{Ac} @$ GMWNT (1), $\mathrm{Mn}_{12} \mathrm{Ac}$ (post-scCO ) (2) and pristine $\mathrm{Mn}_{12}$ Ac. Pellet samples were pressed and wrapped into Teflon film with negligible diamagnetic contribution. Variable-temperature $(1.8-300 \mathrm{~K})$ and field-dependent $(0-5 \mathrm{~T})$ magnetization measurements were carried out using the reciprocating sample option with a sensitivity of $5 \times 10^{-9} \mathrm{emu}$. AC susceptibility measurements were carried out at different frequencies $(1-1,500 \mathrm{~Hz})$ of a $3.5 \mathrm{Oe}$ oscillating field between 1.8 and $10 \mathrm{~K}$.

Methodology used in the magnetic measurements. The investigation of the effects of the encapsulation into nanotube on the magnetic properties of $\mathrm{Mn}_{12} \mathrm{Ac}$ molecules was carried out by comparing the results obtained for $\mathbf{1}$ with those for $\mathbf{2}$ and untreated $\mathrm{Mn}_{12} \mathrm{Ac}$ taking into account the diamagnetic contribution of GMWNT.

Considerations in the magnetic analysis of 1 . The analysis of the magnetic measurements of $\mathbf{1}$ requires the quantification of both the diamagnetic contribution of the GMWNT and the molar concentration of $\mathrm{Mn}_{12} \mathrm{Ac}$ in the sample. To disentangle the effects of the GMWNT on the magnetic properties of the $\mathrm{Mn}_{12} \mathrm{Ac}$ molecules, we first measured the magnetic response of the empty GMWNT. The magnetization loop (Supplementary Fig. S27) shows that GMWNT do not exhibit hysteresis. Moreover, GMWNT have diamagnetic response, because the measured magnetization is negative for positive fields and positive for negative fields. The linear response of $\mathrm{M}$ versus $\mu_{0} H$ allows the determination of the susceptibility $\chi=(-10.0 \pm 0.1) \times 10^{-8} \mathrm{emu} \mathrm{cm}^{-3}$ that is simply given by the slope of the curve.

The susceptibility measurements for GMWNT as function of the temperature are presented in Supplementary Figure S28. The signal from the GMWNT is always negative, that is, diamagnetic (of the order of $-6.38 \times 10^{-6} \mathrm{emu} \mathrm{g}^{-1}$ at $300 \mathrm{~K}$ ) and slightly increases below $42 \mathrm{~K}$ (inset Supplementary Fig. S28). The mass susceptibility of $\mathrm{Mn}_{12} \mathrm{Ac}$ is $2.83 \times 10^{-5} \mathrm{emu} \mathrm{g}^{-1}$ at $300 \mathrm{~K}$ that is $77 \%$ higher than that of the GMWNT. Below $100 \mathrm{~K}$ the difference in mass susceptibility increases further with the $\mathrm{Mn}_{12} \mathrm{Ac}$ reaching $9.03 \times 10^{-3} \mathrm{emu} \mathrm{g}^{-1}$ at $1.8 \mathrm{~K}$ that is three orders of magnitude higher than that of the GMWNT at the same temperature.

The quantification of the molar concentration has been carefully assessed from the magnetic measurements reported in Supplementary Figure S27. $M_{\mathrm{GMWNT}} M_{\mathrm{Mn12Ac}}$ 
and $M_{\mathrm{Mn12A@GMWNT}}$ were defined as the magnetic moments of the GMWNT, $\mathrm{Mn}_{12} \mathrm{Ac}$ and $\mathrm{Mn}_{12} \mathrm{Ac} @ \mathrm{GMWNT}$ samples, respectively. If $m_{\mathrm{GMWNT}}, m_{\mathrm{Mn12Ac}}$ and $m_{\mathrm{Mn} 12 \mathrm{Ac} @ \mathrm{GMWNT}}$ are their respective masses, then the magnetic moments per mass of the GMWNT and $\mathrm{Mn}_{12} \mathrm{Ac}, M_{\mathrm{GMWNT}}^{*}$ and $M_{\mathrm{Mn} 12 \mathrm{Ac}}^{*}$, respectively, can be calculated. As $m_{\mathrm{Mn} 12 @ \mathrm{GMWNT}}=m_{\mathrm{GMWNT}}+m_{\mathrm{Mn12Ac}}$, the following relation holds

$$
M_{\mathrm{Mn}_{12} \mathrm{Ac} @ \mathrm{GMWNT}}=m_{\mathrm{GMWNT}} M_{\mathrm{GMWNT}}^{*}+m_{\mathrm{Mn}_{12} \mathrm{Ac}} M_{\mathrm{Mn}_{12} \mathrm{Ac}}^{*}
$$

Then the mass of $\mathrm{Mn}_{12} \mathrm{Ac}$ and GMWNT can be calculated as

$$
\begin{aligned}
m_{\mathrm{Mn}_{12} \mathrm{Ac}} & =\frac{M_{\mathrm{Mn}_{12} \mathrm{Ac} @ \mathrm{GMWNT}}-m_{\mathrm{Mn}_{12} \mathrm{Ac} @ \mathrm{GMWNT}} M_{\mathrm{GMWNT}}^{*}}{M_{\mathrm{Mn}_{12} \mathrm{Ac}}^{*}-M_{\mathrm{GMWNT}}^{*}} ; \\
m_{\mathrm{GMWNT}} & =m_{\mathrm{Mn}_{12} \mathrm{Ac} @ \mathrm{GMWNT}}-m_{\mathrm{Mn}_{12} \mathrm{Ac}}
\end{aligned}
$$

From the magnetic moment measured at $1.8 \mathrm{~K}$ and $2 \mathrm{~T}$ (Supplementary Fig. S27), it can be deduced that $M_{\mathrm{GMWNT}}^{*}=-0.17 \mathrm{emug}^{-1}, M_{\mathrm{Mn} 12 \mathrm{Ac}}^{*}=37.47 \pm 0.06 \mathrm{emug}^{-1}$ and $M_{\mathrm{Mn} 12 \mathrm{Ac} @ \mathrm{GMWNT}}=0.172 \pm 0.005 \mathrm{emu}$. Because $m_{\mathrm{Mn12A@@GMWNT}}=33.8 \pm 0.1 \mathrm{mg}$, then, $m_{\mathrm{GMWNT}}=29.0$ and $m_{\mathrm{Mn} 12 \mathrm{Ac}}=4.8 \pm 0.7 \mathrm{mg}$.

Magnetoresistance measurements. DC conductivity measurements were performed with the four contacts method for two pressed pellets of each sample: $\mathrm{Mn}_{12}$ Ac@GMWNT (1) and GMWNT (3). Both pellets of each sample gave very similar results demonstrating reproducibility of the measurements. The magnetoresistance measurements were performed in the temperature range $2-300 \mathrm{~K}$ with magnetic fields in the range -8 to $8 \mathrm{~T}$ applied either parallel or perpendicular to the conduction planes. Platinum electrodes $(25 \mu \mathrm{m}$ diameter) were connected to the samples using graphite paste. The samples were measured with cooling and warming rates of $1 \mathrm{~K} \mathrm{~min}^{-1}$ and with DC intensity currents of $0.1-1.000 \mu \mathrm{A}$ in a Quantum Design PPMS-9, giving reproducible results in all cases. Further details of the magnetoresistance measurements can be found in the Supplementary Figs S20-S23.

Quantification of the amount of SMM inside nanotubes. The amount of $\mathrm{Mn}_{12} \mathrm{Ac}$ in $\mathrm{Mn}_{12} \mathrm{Ac} @$ GMWNT (1) can be estimated from TGA (11.6\% wt). From the TGA measurements, the amount of $\mathrm{Mn}_{12} \mathrm{Ac}$ has been deducted by comparing the amount of manganese oxide remaining at $1,000^{\circ} \mathrm{C}$ after all carbon of $\mathrm{Mn}_{12} \mathrm{Ac} @$ GMWNT being burnt out (5.6\% of the total weight, Supplementary Fig. S25) with the residual manganese oxide found at the same temperature for pristine $\mathrm{Mn}_{12} \mathrm{Ac}$ ( $47.2 \%$ of the total weight, Supplementary Fig. S11)

Magnetic measurements described above gave $14.2 \% \mathrm{wt}$ for $\mathrm{Mn}_{12} \mathrm{Ac}$ in $\mathrm{Mn}_{12} \mathrm{Ac} @ \mathrm{GMWNT}$ that is slightly higher than TGA. The accuracy of quantitative SQUID measurements is expected to be greater than that of TGA.

\section{References}

1. Sessoli, R., Gatteschi, D., Caneschi, A. \& Novak, M. A. Magnetic bistability in a metal-ion cluster. Nature 365, 141-143 (1993).

2. Friedman, J. R., Sarachik, M. P., Tejada, J. \& Ziolo, R. Macroscopic measurements of resonant magnetization tunneling in high spin molecules. Phys. Rev. Lett. 76, 3830-3833 (1996)

3. Thomas, L. et al. Macroscopic quantum tunnelling of magnetization in a single crystal of nanomagnets. Nature 383, 145-147 (1996).

4. Ardavan, A. et al. Will spin-relaxation times in molecular magnets permit quantum information processing. Phys. Rev. Lett. 98, 057201 (2007).

5. Schlegel, C., van Slageren, J., Manoli, M., Brechin, E. K. \& Dressel, M. Direct observation of quantum coherence in single-molecule magnets. Phys. Rev. Lett. 101, 147203 (2008).

6. Weiden, N., Kass, H. \& Dinse, K. P. Pulse electron paramagnetic resonande (EPR) and electron-nuclear double resonance (ENDOR) investigation of N@C-60 in polycrystalline C-60. J. Phys. Chem. B 103, 9826-9830 (1999).

7. Dietel, E. et al. Atomic nitrogen encapsulated in fullerenes: effects of cage variations. J. Am. Chem. Soc. 121, 2432-2437 (1999).

8. Morton, J. J. L. et al. Electron spin relaxation of $\mathrm{N} @ \mathrm{C}_{60}$ in $\mathrm{CS}_{2}$. J. Chem. Phys 124, 014508 (2006).

9. Rocha, A. R. et al. Towards molecular spintronics. Nature Mater. 4, 335-339 (2005)

10. Joachim, C., Gimzewski, J. K. \& Aviram, A. Electronics using hybrid-molecular and mono-molecular. Nature 408, 541-548 (2000).

11. Gatteschi, D. \& Sessoli, R. Quantum tunneling of magnetization and related phenomena in molecular materials. Angew. Chem. Int. Ed. 42, 268-297 (2003)

12. Leuenberger, M. N. \& Loss, D. Quantum computing in molecular magnets. Nature 410, 789-793 (2001).

13. Voss, S. et al. Electronic structure of $\mathrm{Mn}_{12}$ derivates on the clean and functionalized Au surface. Phys. Rev. B 75, 045102 (2007).

14. Saywell, A. et al. Self-assembled aggregates formed by single-molecule magnets on a gold surface. Nat. Commun. 1, 75 (2010).

15. Bogani, L. \& Wernsdorfer, W. Molecular spintronics using single-molecule magnets. Nature Mater. 7, 179-186 (2008).
16. Trif, M., Troiani, F., Stepanenko, D. \& Loss, D. Spin-electric coupling in molecular magnets. Phys. Rev. Lett. 101, 217201 (2008).

17. Chamberlain, T. W., Giménez-López, M. C. \& Khlobystov, A. N. Carbon nanotubes as containers: in Carbon Nanotubes and Related Structures: Synthesis, Characterization, Functionalization and Applications (eds Guldi, D.M. \& Martín, N.) 349-384 (Wiley-VCH, 2010)

18. Benjamin, S. C. et al. Towards a fullerene-based quantum computer. J. Phys. Condens. Matter 18, S867-S883 (2006).

19. Bogani, L. et al. Single molecule magnet carbon nanotube hybrids. Angew. Chem. Int. Ed. 48, 746-750 (2009).

20. Kyatskaya, S. et al. Anchoring of rare-earth-based single-molecule magnets on single-walled carbon nanotubes. J. Am. Chem. Soc. 131, 15143-15151 (2009).

21. Giusti, A. et al. Magnetic bistability of individual single-molecule magnets grafted on single-wall carbon nanotubes. Angew. Chem. Int. Ed. 48, 4949-4952 (2009).

22. Ciric, L. et al. $\mathrm{La} @ \mathrm{C}_{82}$ as a spin-active filling of SWCNTs: ESR study of magnetic and photophysical properties. Phys. Status Solidi B 245, 2042-2046 (2008).

23. Khlobystov, A. N. et al. Low temperature assembly of fullerene arrays in single-walled carbon nanotubes using supercritical fluids. J. Mater. Chem. 14, 2852-2857 (2004).

24. Ye, X. R. et al. Supercritical fluid synthesis and characterization of catalytic metal nanoparticles on carbon nanotubes. J. Mater. Chem. 14, 908-913 (2004).

25. Pan, X. \& Bao, X. Reactions over catalysts confined in carbon nanotubes. Chem. Commun. 6271-6281 (2008).

26. Folch, B., Larionova, J., Guari, Y., Guerin, C., Mehdi, A. \& Reye, C. Formation of $\mathrm{Mn}_{3} \mathrm{O}_{4}$ nanoparticles from the cluster $\left[\mathrm{Mn}_{12} \mathrm{O}_{12}\left(\mathrm{C}_{2} \mathrm{H}_{5} \mathrm{COO}\right)_{16}\left(\mathrm{H}_{2} \mathrm{O}\right)_{3}\right]$ anchored to hybrid mesoporous silica. J. Mater. Chem. 14, 2703-2711 (2004).

27. Aubin, S. M. et al. Single-molecule magnets: Jahn-Teller isomerism and the origin of two magnetization relaxation processes in $\mathrm{Mn}_{12}$ complexes. Polyhedron 20, 1139-1145 (2001)

28. Domingo, N. et al. Particle-size dependence of magnetization relaxation in $\mathrm{Mn}_{12}$ crystals. Phys. Rev. B 79, 214404 (2009).

29. van Slageren, J., Dengler, S., Gómez-Segura, J., Ruiz-Molina, D. \& Dressel, M. Magnetism and magnetic resonance studies of single-molecule magnets in polymer matrices. Inorg. Chim. Acta 361, 3714-3717 (2008).

30. de Heer, W. A. et al. Aligned carbon nanotube films: production and optical and electronic properties. Science 268, 845-847 (1995).

31. Kim, G. T. et al. Magnetoresistance of an entangled single-wall carbonnanotube network. Phys. Rev. B 58, 16064-16069 (1998).

32. Cleuziou, J. P., Wernsdorfer, W., Bouchiat, V., Ondarcuhu, T. \& Monthioux, M. Carbon nanotube superconducting quantum interference device. Nature Nanotech. 1, 53-59 (2006).

33. Lis, T. Preparation, structure, and magnetic-properties of a dodecanuclear mixed-valence manganese carboxylate. Acta Crystallogr. Sect. B 36, 2042-2046 (1980)

\section{Acknowledgements}

This work was supported by the Marie Curie Fellowship within the Seventh European Community Framework Programme (M.C.G. and F.M.), UK Engineering and Physical Sciences Research Council, European Science Foundation (A.N.K., J.v.S., A.L.T. and P.D.B), the Royal Society (A.N.K.), Spanish Ministerio de Ciencia y Tecnologia, Generalitat Valenciana (C.J.G.G.) and the Nottingham Nanoscience and Nanotechnology Centre (access to transmission electron microscope). We would like to thank Jose Maria Martinez-Agudo (ICMOL, University of Valencia) for the DSC measurements.

\section{Author contributions}

M.C.G. prepared the materials, developed the experimental methodology, performed the analytical characterization, analysed and interpreted the data. A.L.T. and M.C.G. carried out the $\mathrm{scCO}_{2}$ experiments. A.N.K. conducted the transmission electron microscopy experiments. F.M. and J.v.S. carried out and analysed the magnetic measurements data. C.J.G.G. carried out the magnetoresistance experiments and analysed the data. P.D.B. assisted with the manuscript preparation and analysis of TEM data. M.C.G. and A.N.K. conceived the idea, coordinated the experimental work, discussed the data and wrote the original manuscript. All the authors read and commented on the manuscript.

\section{Additional information}

Supplementary Information accompanies this paper at http://www.nature.com/ naturecommunications

Competing financial interests: The authors declare no competing financial interests.

Reprints and permission information is available online at http://npg.nature.com/ reprintsandpermissions/

How to cite this article: Giménez-López, M.C. et al. Encapsulation of single-molecule magnets in carbon nanotubes. Nat. Commun. 2:407 doi: 10.1038/ncomms1415 (2011). 